\title{
Cognitive performance in high-altitude climbers: a comparative study of saccadic eye movements and neuropsychological tests
}

\author{
Tobias M. Merz • Martina M. Bosch • Daniel Barthelmes • Jacqueline Pichler • \\ Urs Hefti - Kai-Uwe Schmitt • Konrad E. Bloch • Otto D. Schoch • Thomas Hess • \\ Alexander J. Turk $\cdot$ Urs Schwarz
}

Received: 30 November 2012 / Accepted: 23 March 2013/Published online: 6 April 2013

(C) Springer-Verlag Berlin Heidelberg 2013

\begin{abstract}
Impairment of cognitive performance during and after high-altitude climbing has been described in numerous studies and has mostly been attributed to cerebral hypoxia and resulting functional and structural cerebral alterations. To investigate the hypothesis that highaltitude climbing leads to cognitive impairment, we used of neuropsychological tests and measurements of eye movement (EM) performance during different stimulus conditions. The study was conducted in 32 mountaineers participating in an expedition to Muztagh Ata $(7,546 \mathrm{~m})$. Neuropsychological tests comprised figural fluency, line bisection, letter and number cancellation, and a modified pegboard task. Saccadic performance was evaluated under three stimulus conditions with varying degrees of cortical involvement: visually guided pro- and anti-saccades, and visuo-visual interaction. Typical saccade parameters
\end{abstract}

Communicated by Guido Ferretti.

T. M. Merz $(\bowtie)$

Department of Intensive Care Medicine, Bern University

Hospital and University of Bern, Bern, Switzerland

e-mail: tobias.merz@insel.ch

M. M. Bosch · D. Barthelmes

Department of Ophthalmology, University Hospital Zürich,

Zurich, Switzerland

J. Pichler

Department of Pneumology, Bern University Hospital

and University of Bern, Bern, Switzerland

U. Hefti

Swiss Sport Clinic, Stade de Suisse, Sempacherstr. 22,

3014 Bern, Switzerland

K.-U. Schmitt

Institute for Biomedical Engineering, ETH Zürich

and University of Zürich, Zurich, Switzerland (latency, mean sequence, post-saccadic stability, and error rate) were computed off-line. Measurements were taken at a baseline level of $440 \mathrm{~m}$ and at altitudes of 4,497, 5,533, 6,265 , and again at $440 \mathrm{~m}$. All subjects reached 5,533 m, and 28 reached $6,265 \mathrm{~m}$. The neuropsychological test results did not reveal any cognitive impairment. Complete eye movement recordings for all stimulus conditions were obtained in 24 subjects at baseline and at least two altitudes and in 10 subjects at baseline and all altitudes. Measurements of saccade performances showed no dependence on any altitude-related parameter and were well within normal limits. Our data indicates that acclimatized climbers do not seem to suffer from significant cognitive deficits during or after climbs to altitudes above $7,500 \mathrm{~m}$. We demonstrated that investigation of EMs is feasible during high-altitude expeditions.

\section{K. E. Bloch · A. J. Turk}

Pulmonary Division, Department of Internal Medicine,

University Hospital of Zurich, Zurich, Switzerland

O. D. Schoch

Pulmonary Division, Kantonsspital, St. Gallen, Switzerland

T. Hess

Pulmonary Division, Department of Internal Medicine,

Cantonal Hospital, Winterthur, Switzerland

U. Schwarz

Department of Neurology, University Hospital Zürich,

Zurich, Switzerland 
Keywords High altitude $\cdot$ Hypoxia $\cdot$ Saccadic eye movement $\cdot$ Neuropsychological testing $\cdot$ Cognitive function

\section{Introduction}

An ever-increasing number of recreational climbers attempt ascents of summits of extreme altitudes. The Nepalese Ministry of Tourism and Civil Aviation and the Nepal Mountaineering Association reported having issued permits to 6,032 climbers for peaks higher than $6,000 \mathrm{~m}$ in the year 2010 alone. Summits of very high altitude exist and are also often climbed in other parts of the Himalayas and in South America and Alaska.

Alterations in cognitive performance during and after high-altitude climbing have been described in numerous reports (Lieberman et al. 1995; Abraini et al. 1998) and have mostly been attributed to cerebral hypoxia caused by low atmospheric oxygen partial pressure and to functional cerebral impairment due to acute mountain sickness (AMS) or high-altitude cerebral edema (HACE) (Virues-Ortega et al. 2004). The possibility of long-term cerebral damage and impairment from exposure to extreme hypoxia and the resulting hypoxemia has been a topic of controversy for decades (West 1986). Several investigators have found persistent neuropsychological deficits in high-altitude climbers (Hornbein et al. 1989; Regard et al. 1989). Other authors have documented persisting structural cerebral changes with magnetic resonance imaging (MRI) (Fayed et al. 2006; Garrido et al. 1993) after high-altitude climbs. Fayed et al. (2006) reported brain lesions in mountain climbers detected by means of conventional MRI and magnetic resonance spectroscopy (MRS) after an ascent to relatively moderate altitudes of 4,800 $\mathrm{m}$ in the French Alps (Fayed et al. 2006). In contrast to these studies, other investigators did not observe any significant changes either in brain imaging results (Anooshiravani et al. 1999) or in neuropsychological testing (Jason et al. 1989) after expeditions to heights between 5,334 m (high altitude) and 7,500 m (extreme altitude).

A possible explanation for these ambiguous findings is that most neuropsychological tests do not discriminate between cognitive impairment due to neuronal dysfunction and behavioral changes caused by other factors such as fatigue, mood fluctuations and lack of motivation (Nicolas et al. 2000). The overall reliability of many experimental results may have been affected by the small number of subjects included in most previous studies, the inter-subject variability inherent to standard neuropsychological testing and the difficulty of carrying out more complex neurological examinations at high altitudes.
The aim of the prospective cohort study at hand was to evaluate cognitive functions in a large group of climbers before, during, and after an expedition to high altitudes, independent of the above-mentioned confounding factors. Prerequisite for the applied method was a high sensitivity for detection of deterioration of cerebral function, a high inter-rater and test-retest reliability, and a low intra-individual variation of baseline measurements in normoxic conditions. For this purpose, we recorded visually elicited saccades in mountain climbers and compared their results with those obtained from an array of traditional neuropsychological tests. Saccadic eye movements, which very quickly and accurately change the eye's position, have been studied for decades in both humans and animals. The various networks involved in their control have been localized and characterized by numerous experiments in animals using single cell recordings, as well as by behavioral investigations in animals, normal subjects, and patients using eye movement recordings, or functional studies using magnetic resonance imaging (fMRI) or transcranial magnetic stimulation (TMS) (Leigh and Kennard 2004). The fact that eye movements can be recorded at a very high temporal and spatial resolution (Collewijn et al. 1975) has been of particular benefit for investigators since reliable parameters of brain function can be deduced from such eye position tracks. Depending on the stimulus condition, these parameters may help to distinguish pathological conditions such as muscular/neuromuscular fatigue (Barton et al. 1995) from various cortical and/or subcortical dysfunctions, including lack of attention (Gaymard et al. 1998; Pierrot-Deseilligny et al. 1991) as well as motivation.

The decline in saccadic eye movement performance is a sensitive marker even for minor impairment of cerebral function in various physiological and pathological conditions. Sleep deprivation for $24 \mathrm{~h}$ has been reported to impair peak velocity of saccades and to lead to deficits of accuracy and latency (Fransson et al. 2008). As compared to placebo, low-dose lorazepam (1-2 mg) significantly increases saccade latency and decreases peak velocity (Masson et al. 2000). Similar effects can be seen after consumption of moderate amounts of ethanol (Vorstius et al. 2008). In addition, specific and marked impairment of saccadic eye movements have been reported in early Alzheimer's (Yang et al. 2012), mild frontotemporal dementia (Boxer et al. 2006), and in post concussion syndrome (Heitger et al. 2008). To our knowledge, this is the first report on saccadic eye movements in the context of prolonged hypobaric hypoxia. 


\section{Methods}

\section{Objectives}

To evaluate cognitive functions in a large group of climbers before, during, and after exposure to hypobaric hypoxia in the context of an expedition to extreme altitudes.

\section{Participants}

Thirty-two experienced non-professional mountaineers (7 females, 25 males) with a mean age of 43 years (range 25-62 years) participated in The Swiss High-Altitude Research Expedition Muztagh Ata carried out on Mount Muztagh Ata $(7,546 \mathrm{~m})$ in western China. All subjects were lowland residents residing below 1,400 m. Exclusion criteria were cardiac and/or respiratory diseases, regular medication, previous history of high-altitude pulmonary edema after a rapid ascent ( $<3$ nights) at altitudes below $3,500 \mathrm{~m}$, and previous history of severe AMS or highaltitude cerebral edema after rapid ascent to altitudes below $3,500 \mathrm{~m}$.

\section{Altitude measurements}

The subjects were randomly assigned to two groups ascending at slightly different rates. Each group consisted of 16 mountaineers, who were to climb from $3,750 \mathrm{~m}$ (day 1 of expedition) to the base camp ( $\mathrm{BC}=4,497 \mathrm{~m})$, camp 1 $(\mathrm{C} 1=5,533 \mathrm{~m}), \quad$ camp $2(\mathrm{C} 2=6,265 \mathrm{~m}), \quad$ camp 3 $(\mathrm{C} 3=6,865 \mathrm{~m})$ and to the summit $(7,546 \mathrm{~m})$. Daily assessment of symptoms and signs of AMS and high-altitude pulmonary edema (HAPE) was performed. AMS was determined by means of the Lake Louise AMS protocol and the cerebral score on the environmental symptoms questionnaire (AMS-C score) (Sampson et al. 1983). The Lake Louise Protocol includes a self-reported questionnaire in combination with a clinical assessment. The questionnaire involves individual subject ratings of headache, gastrointestinal symptoms, fatigue, dizziness, and difficulty in sleeping [individual scores from 0 (absence of symptoms) through 3 (severe symptoms); overall subject score 0 (no signs of AMS) through 15 (maximum)]. For the clinical assessment, subjects were examined for changes in mental status, ataxia, and peripheral edema. Changes in mental status and ataxia (heel-to-toe walking test) were rated from 0 (no signs) to 4 (coma) points, while peripheral edema was rated as 0 points (no edema), one point (peripheral edema present in one location) or two points (peripheral edema present in more than one location). The Lake Louise AMS score (LLS) was calculated by adding the scores of all eight items: a score $\geq 5$ defined the presence of AMS. To obtain the AMS-C score, the subject was asked eleven questions about the following symptoms: light-headedness, headache, dizziness, dim vision, difficulties with coordination, gastrointestinal upset, hangover sensation and loss of appetite, and weakness; points were assigned from 0 (no symptoms at all) to 5 (extreme symptoms). The average value of the severity of each symptom in the group was used to calculate the mean score of cerebral (AMS-C), scores greater than 0.7 defined the presence of AMS. The individual mean arterial oxygen saturation $\left(\mathrm{SaO}_{2}\right)$ and the LLS and AMS-C scores for each subject and every altitude (BC, C1, C2, C3) were calculated and used for further analysis. Heart rate and arterial $\mathrm{SaO}_{2}$ were measured by finger pulse oximetry (Onyx 9500 SportStat, Nonin Medical Inc., Plymouth MN, USA). This pulse oximeter has a three-color pulse-quality indicator and guarantees an accuracy of $\pm 2 \%$ within the range of $70-100 \% \mathrm{SaO}_{2}$. The climbers warmed their hands prior to each measurement to ensure good reading quality. Each expedition member kept a diary on his location in the morning and the evening, the maximally reached height during the day, physical activity level, and potential drug intake. Intake of medication other than NSAIDS was allowed only upon recommendation by the expedition physician. Altitude measurements for eye movement recordings and neuropsychological testing were performed at $\mathrm{BC}, \mathrm{C} 1$, and $\mathrm{C} 2$. Measurements were performed on the day following the first night after reaching a new altitude. No physical activity was allowed during the test days. Measurements were performed at the same time of the day for each subject in dedicated high-altitude study sites. These consisted of large tents that allowed for comfortable testing conditions and provided sufficient protection from wind and UV-radiation. Temperatures inside the research tents ranged from 10 to $25^{\circ} \mathrm{C}$. Unlimited amounts of food and fluids were provided by the expedition's auxiliary staff. Coffee but no other stimulant was provided during morning hours, alcohol was not available throughout the expedition.

\section{Measurement of saccadic eye movement}

Eye movement recordings were performed prior to the expedition, in Zürich (ZH1, $440 \mathrm{~m}$ ), and at $\mathrm{BC}, \mathrm{C} 1$, and $\mathrm{C} 2$; all tests were repeated in Zürich (ZH2) 3 months after the expedition. Subjects were familiarized with the eye movement tasks during one session at the time of $\mathrm{ZH} 1$. Subjects were seated comfortably at a table with a soft chin-rest. The right eye position was precisely measured using a novel, video-based infrared (IR) eye tracker (OCULOMETRICS, Zurich, Switzerland) with a sampling rate of $500 \mathrm{~Hz}$ and a spatial resolution of $0.1^{\circ}$, which is comparable to the traditional magnetic search coil technique (Collewijn et al. 1975). If eye movement traces were 
contaminated by blinks, this was detected by the videobased eye tracker software and the traces were excluded from all further analyses. After each recording session, eye movement traces were evaluated by tracking both the pupil and the limbus. Tracking was performed by first determining the center of the pupil using a modified circular Hough transformer, and, by detecting the left and right pupil edge as well as the left and right border of the iris using an adaptive edge detection algorithm (Schmitt et al. 2007). Finally, raw IR signals were stored for off-line analysis. Stimuli were presented on an 18-inch LCD monitor (Belinea 101920, 1,280 × 1,024 pixels, $376 \times 301 \mathrm{~mm}, 60 \mathrm{~Hz}$ ), which was positioned $490 \mathrm{~mm}$ from the right, center-aligned eye (visual area subtending $\pm 37^{\circ}$ horizontally and $\pm 31^{\circ}$ vertically). In addition, a piece of black cardboard with only a small slit was positioned close to the subject's face to prevent inadvertent influences from the visual surroundings. Each trial started with a period of fixation $(500 \mathrm{~ms})$ on a small center target $\left(0.8 \times 0.8^{\circ}\right)$. After successful fixation, the center target disappeared and a new stimulus was presented for $800 \mathrm{~ms}$. Subsequently, the trial ended with a dark screen of $200 \mathrm{~ms}$ duration. For each of the three visual paradigms, 126 trials were recorded in a block during a brief session of $\sim 3 \mathrm{~min}$. Because of the time needed to compute and store the raw tracking data, a complete test run lasted $\sim 30 \mathrm{~min}$. Three different paradigms were used in the same sequence during each session at each altitude (Fig. 1):

1. Visually guided pro-saccades (PRO) were used to assess the state of simple, reflexive eye movements. The target was presented on a black background at $5^{\circ}$, $10^{\circ}, 15^{\circ}$, and $20^{\circ}$ eccentricity rightwards and leftwards with the same randomized sequence for all sessions and subjects.

2. Visually guided, voluntary anti-saccades (ANTI) were used to assess the ability of frontal neural networks, including their inter-hemispheric connections, to suppress reflexive saccades (top-down task) (Munoz and Everling 2004). Targets were presented as in the prosaccade task, but the subject was required to gaze at a virtual target in the horizontal mirror position.

3. The visuo-visual interaction paradigm (VVI) was used to evaluate the performance of extrastriate parietal brain areas during complex visual stimulation including optic flow patterns (Ilg et al. 2006; Schwarz et al. 2010). Targets were presented in the same eccentricities as in the pro-saccade task but on a background consisting of 300 randomly distributed line elements $\left(0.2 \times 0.8^{\circ}\right)$, which was moved downwards for $200 \mathrm{~ms}$ at a speed of $11 \%$ s at the onset of the trial (i.e., motion began when the target was presented in a new location).
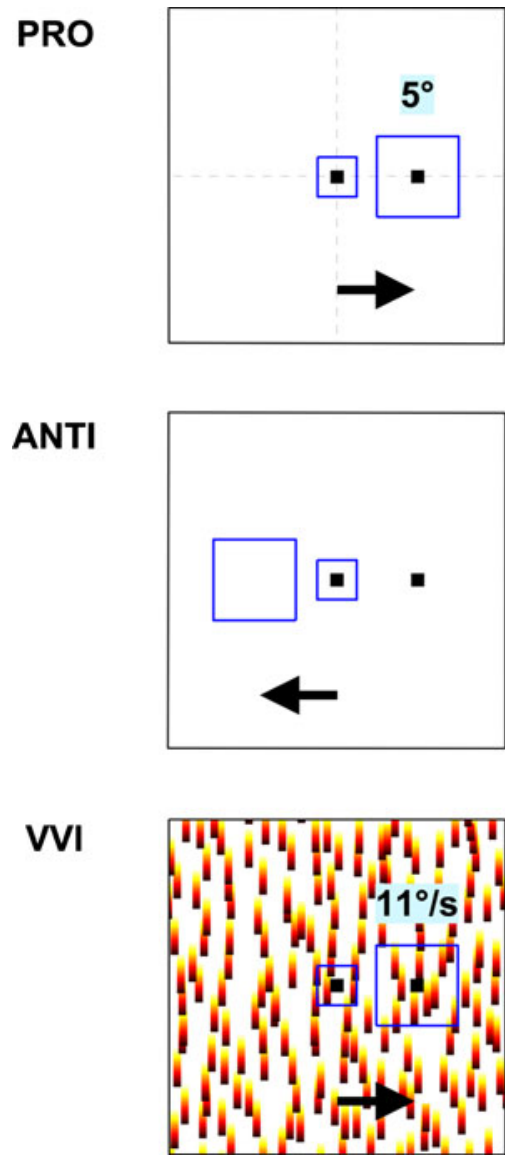

Fig. 1 The three visual paradigms used in this study (see text for explanations). Visually guided pro-saccades (top panel), visually guided, voluntary anti-saccades (middle panel), visuo-visual interaction (bottom panel). Arrow direction of required eye movement

Neuropsychological testing

Neuropsychological testing was performed prior to the expedition in Zürich (ZH1, $440 \mathrm{~m}$ ) and, subsequently, at $\mathrm{BC}, \mathrm{C} 1$, and $\mathrm{C} 2$; all tests were repeated in Zürich 3 months after the expedition. Subjects were familiarized with the applied neuropsychological tests by performing a trial run for each test at the time of ZH1. An array of four neuropsychological tests was used to evaluate cognitive functions:

1. The line bisection test (LB) (Schenkenberg et al. 1980) involves neural circuits of the prefrontal cortex, the parietal lobes, and several visual processing areas bilaterally, as well as the cerebellar vermis (Fischer 2001). It consists of 12 black horizontal lines of 100-200 mm length on a sheet of white A4 paper. Each subject had to mark the center of every line using his or her dominant hand. The deviation of the attempted bisection from the true center of each line was calculated as the percentage of the deviation from 
the mark to the midpoint (Braun and Kirk 1999). An overall mean PD of $\leq 5 \%$ was considered normal.

$\mathrm{PD}_{\text {score }}=100 \% \times \frac{\Delta \text { midpoint }[\mathrm{mm}]}{\text { length of line }[\mathrm{mm}]}$

2. The 2/7 cancellation test was used to assess verbal fluency, a measure for bilateral and left frontal brain regions. The subject was given a sheet of paper with a series of lines consisting of either letters or numbers in which the target numbers 2 and 7 were embedded (Ruff et al. 1992). In the numeric condition, the numbers 2 and 7 were randomly interspersed with other numbers ( $2 / 7$ numbers). In the letter condition, distracters were letters of the alphabet (2/7 letters). The test required the subject to cross out the specific targets (always the numbers 2 and 7) as quickly as possible. Outcome measures were the time span to complete the task and the number of errors (missed target digits or crossed out distracters). Five different versions of the $2 / 7$ test were available and applied in a prerandomized order to avoid a learning effect.

3. An applied modified version of the Ruff figural fluency test (RFFT) was used to evaluate non-verbal fluency, which predominantly employs functions of the right frontal lobe (Ruff et al. 1994). Subjects had to generate different unique designs using straight lines to connect five dots embedded in a square. The five-dot stimuli were presented in 35 contiguous templates, which were arranged in a five-square-by-seven-square grid. Evaluated parameters were the time to complete the task, the number of design repetitions, and the number of incomplete designs after expiration of the time limit of 3 min.

4. A modified Pegboard test, the Chess test (CT), was used to globally assess visuo-motor skills. Subjects had to select chess figures from a covered box and correctly position them on a small chessboard $(150 \times 150 \mathrm{~mm})$. For each session, one of four possible pre-randomized positions of the chessboard (rotation of $0-270^{\circ}$ in steps of $90^{\circ}$ ) was used. Outcome measures were time to complete the task and number of misplaced chess figures.

Data analysis for measurement of saccadic eye movement

All data processing was performed off-line using a commercial software package (MATLAB 7.6, The MathWorks Inc., Natick, MA). First, eye position was filtered using an adaptive smoothing cubic spline. Then, eye velocity was obtained by a two-point differentiation. Finally, saccades were detected automatically using combined velocity and acceleration criteria (Schwarz and Miles 1991). For each paradigm and eccentricity (with rightward and leftward saccades combined), the latency (time between target movement and saccade onset), post-saccadic accuracy (difference between target and eye position over an interval of $50 \mathrm{~ms}$ ), and the saccade main sequence (Lebedev et al. 1996; Becker 1989), as well as the number of correct trials, were determined. The saccadic main sequence denotes the relationship between the peak velocity of the eye movement and the displacement as follows:

$V_{\text {peak }}=V_{0} \times\left[1-\exp \left(\frac{-\Delta \text { eye pos }}{\tau}\right)\right]$

$V_{0}$ the saturation velocity, $\tau$ amplitude for which peak velocity reaches $63 \%$ of $V_{0}$.

Due to missing data, subjects were analyzed in three subgroups (S1 to S3) (Table 2). S1 comprises all subjects participating in the study. S2 includes subjects who were evaluated in $\mathrm{ZH} 1$ and $\mathrm{ZH} 2$ and on at least two altitudes. A one-way analysis of variance (ANOVA) followed by a post hoc test (Scheffé) was applied to their data. Finally, S3 includes only subjects for whom all 15 recordings were available, and for whom a repeated measures ANOVA was therefore applicable (StatView 5.0.1, SAS Institute Inc., Cary, NC). The use of significance level of $p<0.01$ is a standard in the field of visuo-motor research, thus a $p$ value of $<0.01$ was considered significant for all eye movement statistics.

Data analysis for neuropsychological testing

A mixed design analysis of variance which accounts for missing values, was applied to the results of the neuropsychological tests (mean $\pm \mathrm{SD}$ ) at different altitudes, followed by a multiple comparison test (Scheffé test). Within-subject factors were $\mathrm{SO}_{2}, \mathrm{LLs}$, and AMS-c, respectively. A $p$ value of $<0.05$ was considered statistically significant.

\section{Ethics approval}

The study was approved by the Ethics Committee of the University Hospital of Zürich and adhered to the tenets of the Declaration of Helsinki. Informed, written consent was obtained from all participants.

\section{Results}

All of the 32 subjects in the present study reached $\mathrm{C} 1$, and 28 reached $\mathrm{C} 2$. $\mathrm{C} 3$ was reached by 24 climbers and the summit by 17 . Two participants were not allowed to climb 
Table 1 Oxygen saturation and mountain sickness at different altitudes

\begin{tabular}{llllll}
\hline & $\mathrm{BC}$ & $\mathrm{C} 1$ & $\mathrm{C} 2$ & $\mathrm{C} 3$ & $p$ \\
\hline $\mathrm{SaO}_{2}(\%)$ & $83.5 \pm 2.8$ & $77.9 \pm 4.5$ & $74.7 \pm 5.7$ & $70.0 \pm 6.0$ & $<0.0001$ \\
$\begin{array}{c}\mathrm{LLs} \\
\text { (points) }\end{array}$ & $2.2 \pm 1.0$ & $2.9 \pm 1.6$ & $3.3 \pm 1.7$ & $3.5 \pm 2.3$ & 0.015 \\
$\begin{array}{c}\mathrm{AMS}-\mathrm{c} \\
\text { (points) }\end{array}$ & $0.1 \pm 0.1$ & $0.2 \pm 0.2$ & $0.3 \pm 0.4$ & $1.69 \pm 2.09$ & $\mathrm{Ns}$ \\
\hline
\end{tabular}

LLs acute mountain sickness (AMS) assessed by applying the Lake Louise AMS protocol; AMS-c AMS assessed by applying the cerebral score of the environmental symptoms questionnaire; $B C$ base camp (4,497 m); $C 1$ camp 1 $(5,533 \mathrm{~m}) ; \mathrm{C} 2$, camp $2(6,265 \mathrm{~m}) ; \mathrm{SaO}_{2}$ arterial oxygen saturation measured by pulse oximetry; $n s$ not significant

beyond 5,400 m, one due to severe mountain sickness and lack of physical fitness, and the other due to insufficient skiing skills. Two subjects chose to abort the climb between $\mathrm{C} 1$ and $\mathrm{C} 2$, and four subjects between $\mathrm{C} 2$ and $\mathrm{C} 3$ because of exhaustion and lack of motivation. The two groups reached the summit at 7,546 m within 20-21 days. From the start of the climb at $3,750 \mathrm{~m}$, the mean ascent rate was $191-201 \mathrm{~m} / \mathrm{d}$. Table 1 shows individual mean $\mathrm{SaO}_{2}$ for each altitude at which data were obtained. $\mathrm{SaO}_{2}$ significantly decreased at higher altitudes, whereas the individual mean LLS and mean AMS-C scores of the expedition members showed a trend toward decrease, but no significant differences. Depending on the scoring system applied, 29 of 32 subjects (90.6\%) (LLS $>5$ ) or 22 of 32 subjects $(68.7 \%)$ (AMS-C $>0.7)$ experienced at least one episode of AMS during the course of the expedition. LLS and AMS-C scores correlated significantly (Spearman correlation coefficient $=0.6226, p<0.001$ ).

\section{Eye movement experiments}

Figure 2 is a representative sample showing the main sequence and the latency distribution of horizontal eye positions sampled during the VVI paradigm of a study subject at $\mathrm{C} 1$. The corresponding computation of the typical saccade parameters from the eye movement traces is shown in Fig. 3. No difference in either saccade latency or main sequence at different altitudes was found in our subjects. Figure 4 is an example of latency results from the one-way ANOVA with post hoc test (multiple comparisons) for the same subject. The data clearly show the tight clustering around typical means (PRO $171 \pm 9 \mathrm{~ms}$, ANTI $263 \pm 15 \mathrm{~ms}$, VVI $250 \pm 10 \mathrm{~ms}$ ) at all altitudes. Only the ANTI latencies at BC were statistically different when compared to the $\mathrm{ZH} 2$ results $(p<0.01)$. Table 2 lists for all recordings the percentage of such significant differences observed in a particular session, which was very small for all subgroups.
In the group S1 comprising of all subjects participating in the study no distinct differences in the main parameters compared to S2 and S3 were found. The subject subgroup S2 comprised of twenty-four (4 females, 20 males) climbers who were evaluated in $\mathrm{ZH} 1$ and $\mathrm{ZH} 2$ and on at least two altitudes. This group was used to examine possible dependencies of the eye movements on altitude. In order to look for inter-individual trends, all data were normalized with respect to the mean of the $\mathrm{ZH} 2$ results. Although there were apparent weak trends in both, latency (slightly shorter at higher altitudes, Fig. 5) and the main sequence (slightly faster at higher altitudes, Fig. 6), the results were statistically not significant (note the narrow window of only \pm 0.2 , which was necessary to illustrate these trends). Figure 7 shows the percentage of correct trials for the different paradigms at different altitudes. All differences were consistent and similar for all altitudes. A one-way ANOVA with post hoc test was applied for latencies and main sequences for all three paradigms. All grand mean values were within normal ranges of the respective task (latency: PRO $179 \mathrm{~ms}$, ANTI $264 \mathrm{~ms}$, and VVI $270 \mathrm{~ms}$; main sequence: PRO 548\%, ANTI 466\%, VVI $552 \%$ s). Again, there were no significant parameter differences with respect to altitude.

The subgroup $\mathrm{S} 3$ comprised of ten subjects ( 2 females, 8 males) with complete data sets for every paradigm at every altitude, and was used to confirm the above results using a traditional repeated measures ANOVA. As expected, the procedure revealed a significant effect of the factor paradigm (latency: $p<0.0001$, main sequence: $p<0.0013$ ) but not of the factor altitude (latency: $p=0.0449$, main sequence: $p=0.0230$ ). Similar to saccadic latency and the main sequence, post-saccadic accuracy was independent of the altitude and entirely within the normal range. Furthermore, a thorough visual inspection of all saccades did not reveal any potential pathology, such as catch-up saccades, post-saccadic glissades, and/or post-saccadic drifts.

\section{Neuropsychological experiments}

Data was recorded for 32 subjects on all altitudes from $\mathrm{ZH} 1$ to $\mathrm{C} 1$, and in 24 subjects on all altitudes from $\mathrm{ZH} 1$ to C2. Data in $\mathrm{ZH} 2$ was again recorded in all 32 subjects.

Table 3 shows the results of the different neuropsychological tests with respect to altitude, $\mathrm{SaO}_{2}$, and degree of AMS expressed by LLS and AMS-c. In the LB test the subjects showed a mean PD of $2.5 \pm 0.7 \%$ in $\mathrm{ZH} 1$, and the altitude measurements at $\mathrm{BC}, \mathrm{C} 1$, and $\mathrm{C} 2$ yielded no significant changes. After the expedition, PD was $1.9 \pm 0.8 \%$, resulting in a significant overall decrease of PD $(p<0.001)$. None of the subjects showed a PD $\geq 5 \%$ on any measurement. LB results were not correlated with either $\mathrm{SaO}_{2}$ or AMS scores. The time needed to complete 
S12 VVI C1 (5533m)
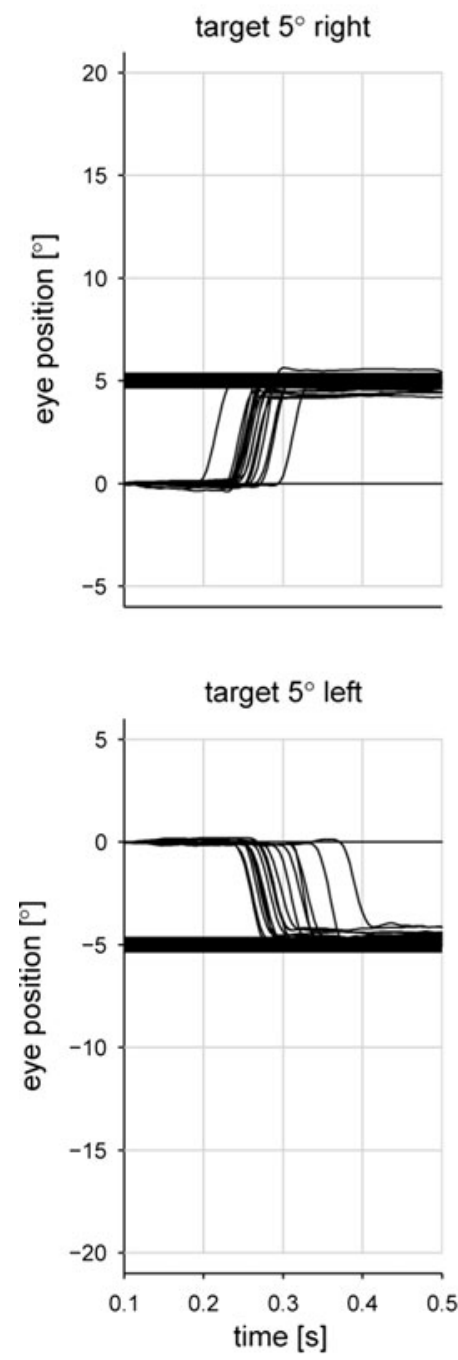
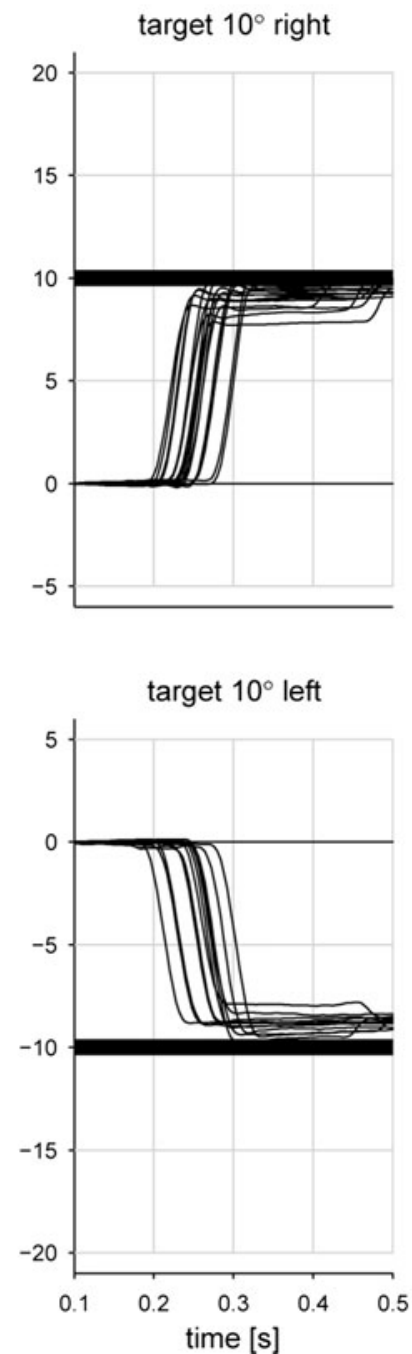
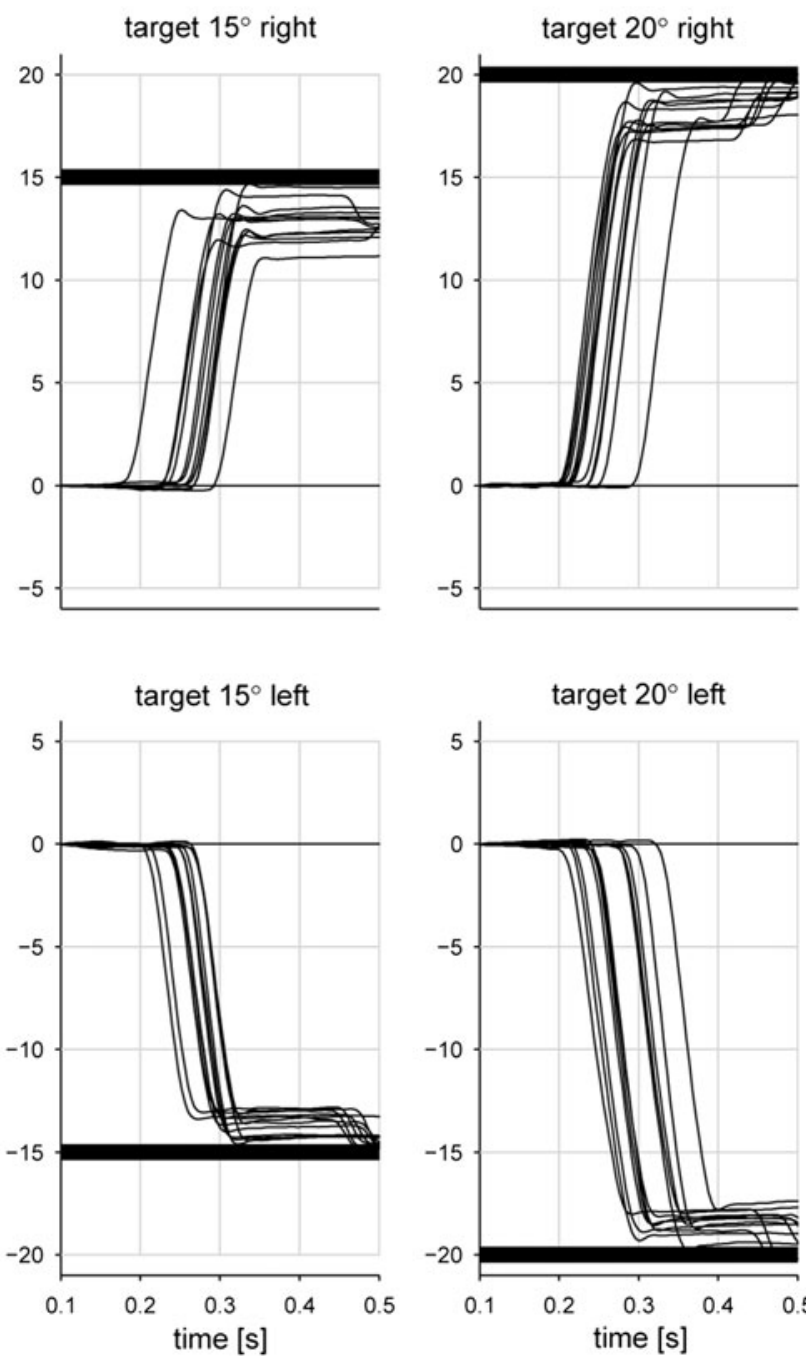

Fig. 2 Main sequence and latency distribution of horizontal eye positions sampled during the VVI paradigm of one subject at C1. Note postsaccadic accuracy and stability for all eccentricities

the $2 / 7$ numbers and the amount of errors showed no correlation with altitude, $\mathrm{SaO}_{2}$ or AMS scores. The amount of time required to complete the $2 / 7$ letters decreased significantly, from $66.1 \pm 16.9 \mathrm{~s}(\mathrm{ZH} 1)$ to $52.7 \pm 22.1 \mathrm{~s}(\mathrm{C} 2)$, and displayed a non-significant increase to $55.2 \pm 16.6 \mathrm{~s}$ in ZH2; accordingly, we found a significant negative correlation with AMS-c but not with LLS or $\mathrm{SaO}_{2}$. No correlation existed between error amount and altitude, $\mathrm{SaO}_{2}$ or AMS scores. The time required to complete the RFFT significantly decreased from $132.1 \pm 32.8 \mathrm{~s}$ in $\mathrm{ZH} 1$ to $96.9 \pm 40.2 \mathrm{~s}$ at $\mathrm{C} 2$, and increased to $106.2 \pm 42.1 \mathrm{~s}$ in $\mathrm{ZH} 2$. There was a negative correlation with AMS-C, whereas the amount of repetitions was not correlated with altitude, $\mathrm{SaO}_{2}$ or AMS scores. The time to complete the CT revealed no significant correlations as well.

\section{Discussion}

The findings of our study can be summarized as follows: the well-acclimatized climbers generally did not suffer from significant effects of hypoxia on their cognitive performance during and after a prolonged stay at very high altitudes. The behavior of eye movements was in close agreement with the results of traditional neuropsychological tests. It was possible to reliably record eye movements during a high-altitude expedition for the first time.

Saccadic performance has been reported to be a sensitive marker even for minor impairment of cerebral performance in various physiological and pathological conditions (Green and King 1998; Heitger et al. 2008; Yang et al. 2012). Saccadic eye movement studies offer a 

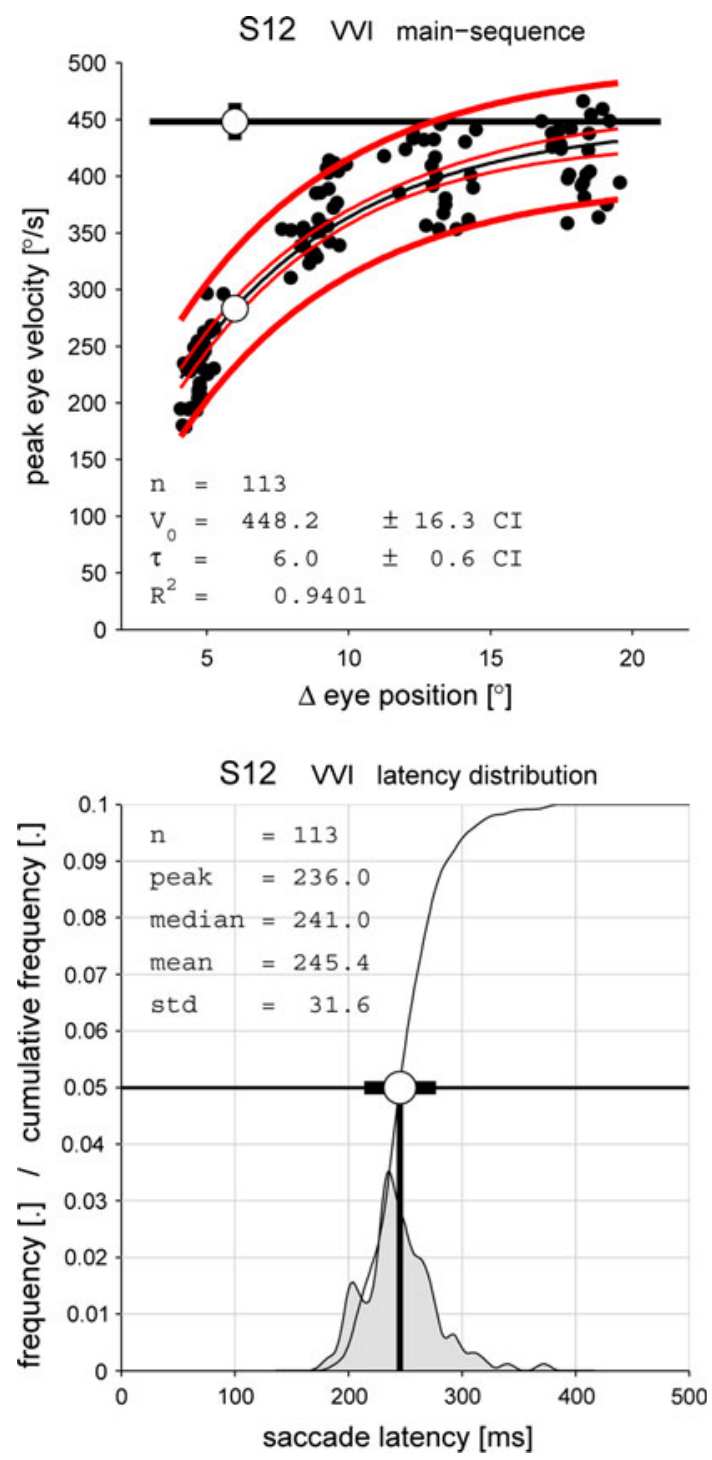

Fig. 3 Computation of typical saccade parameters from the eye movement traces shown in Fig. 2. Main sequence (top panel) with saturation velocity $\left(V_{0}\right)$ and velocity at $63 \%(\tau)$ shown as white circles, CI confidence intervals; latency distribution (bottom panel) with mean $\pm \mathrm{SD}$ shown as white circle and bold lines. Note cumulative frequency was divided by 10 to fit it into the axes

number of advantages in the evaluation of cerebral performance and possible impairment in the context to prolonged exposure to hypoxic conditions at high altitudes. The experiments measure physiological data without human interaction during the recording period and thus exclude observer-related bias. In addition, the stimulus paradigms and recording times can easily be adjusted to the goal of the study. The different yet consistent patterns that were obtained from the saccadic parameters in our subjects reveal that the responses to the three visual paradigms were mediated by different cortical networks-including all known cortical/subcortical and infratentorial optomotor
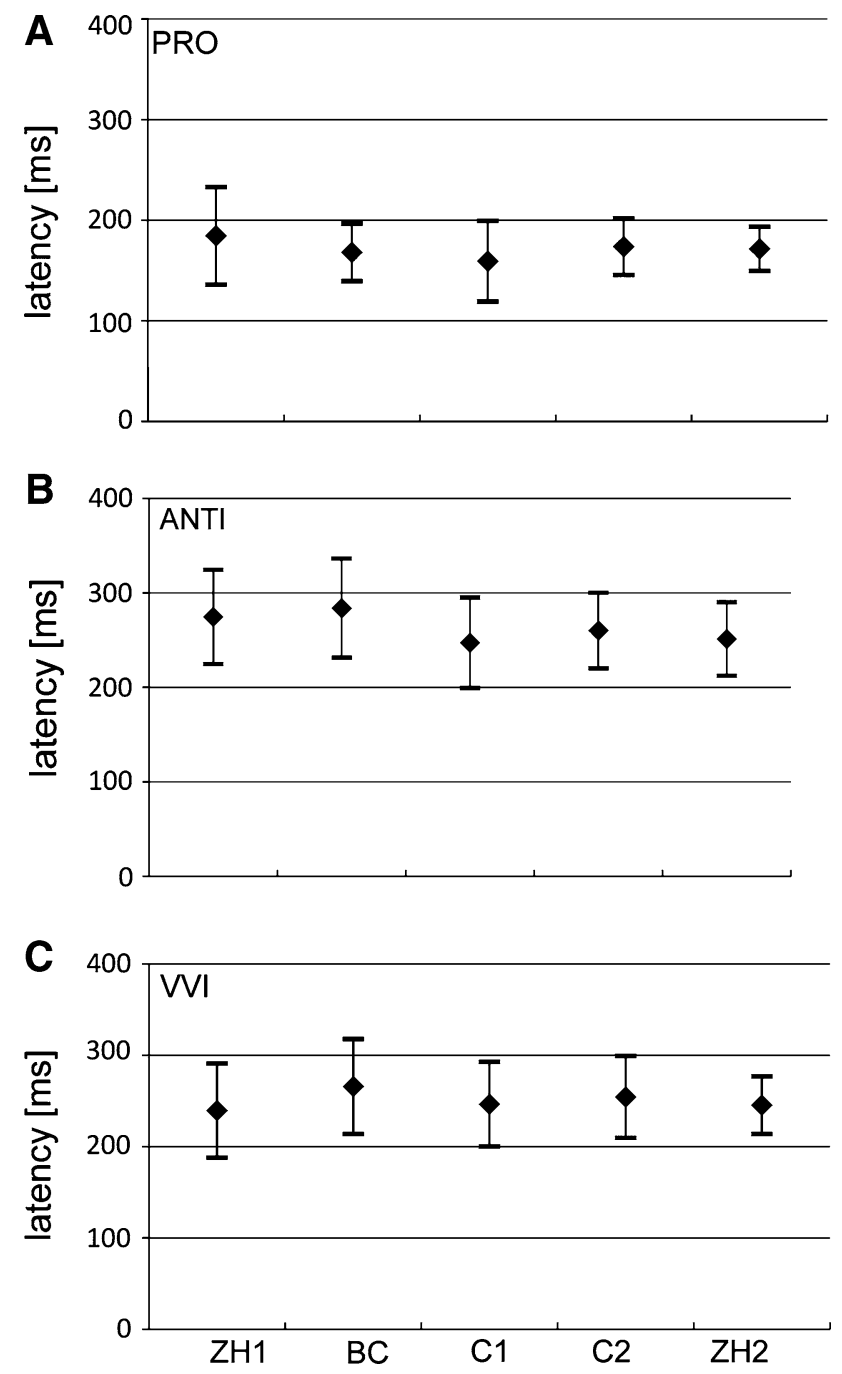

Fig. 4 One-way ANOVA with post hoc test (Scheffé) for all latencies of subject 12. The number of trials for each condition is shown at the bottom of each axis

areas - and would have allowed easy detection of performance impairment. There were a small number of statistically significant differences in the results. However, the aberrations were too small and random to indicate a true and systematic optomotor deficit, since such failures would produce a more dramatic, persistent, and well-known pathological pattern in the eye movement parameters. Muscular and/or neuromuscular fatigue would result in a pattern consisting of more and more catch-up saccades and glissades (Bahill and Stark 1975), and/or the saturation velocity would significantly decrease during the strenuous 9 min of forced eye movements, as is observed in ocular myasthenia (Barton et al. 1995; Spooner and Baloh 1979). Cortical deficits exhibit typical patterns depending on the lesion site. Even a partial breakdown of a neural network results in either missing a chain of neurons with wellknown transmission times (e.g., appearance of express 
Table 2 Statistical results for three subgroups of subjects

\begin{tabular}{|c|c|c|c|c|c|c|c|c|c|}
\hline \multirow[t]{3}{*}{ Subjects } & \multirow[t]{3}{*}{ Sessions } & \multirow[t]{3}{*}{ APH } & \multirow[t]{3}{*}{ Trials } & \multicolumn{6}{|c|}{ Stimulus condition } \\
\hline & & & & \multicolumn{2}{|l|}{ PRO } & \multicolumn{2}{|l|}{ ANTI } & \multicolumn{2}{|l|}{ VVI } \\
\hline & & & & $V_{0}(\%)$ & Latency (ms) & $V_{0}(\%)$ & $\overline{\text { Latency }(\mathrm{ms})}$ & $V_{0}(\%)$ & $\overline{\text { Latency }(\mathrm{ms})}$ \\
\hline Subgroup $1(n=32)$ & 396 & 6.6 & 31,061 & $539 \pm 72$ & $184 \pm 18$ & $453 \pm 75$ & $271 \pm 31$ & $550 \pm 76$ & $271 \pm 25$ \\
\hline Subgroup $2(n=24)$ & 315 & 5.7 & 25,308 & $548 \pm 71$ & $180 \pm 18$ & $469 \pm 80$ & $266 \pm 32$ & $559 \pm 75$ & $271 \pm 27$ \\
\hline Subgroup $3(n=10)$ & 150 & 4.0 & 12,052 & $536 \pm 60$ & $179 \pm 21$ & $453 \pm 81$ & $270 \pm 28$ & $544 \pm 54$ & $268 \pm 21$ \\
\hline
\end{tabular}

Note the similarity for all data across the three subgroups and the small percentage of significantly different results

Subjects number of subjects in each subgroup. 1 all subjects; 2 subjects with at least two recording sessions in $\mathrm{BC}, \mathrm{C} 1$, or $\mathrm{C} 2$, and at least one session in $\mathrm{ZH} 1$ or $\mathrm{ZH} 2$ for each stimulus condition; 3 subjects with a full set of 15 recording sessions, which were used for the repeated measures ANOVA. APH percentage of sessions with results which were significantly different $(p>0.01)$ in the ANOVA post hoc test. Trials number of valid saccades. Values are grand mean \pm SD for the mean data of each subject's recording session. $V_{0}$ asymptotic limit for the maximum speed of the main sequence (see text). Stimulus conditions: PRO visually guided pro-saccades; $A N T I$ anti-saccades; VVI visuo-visual interaction
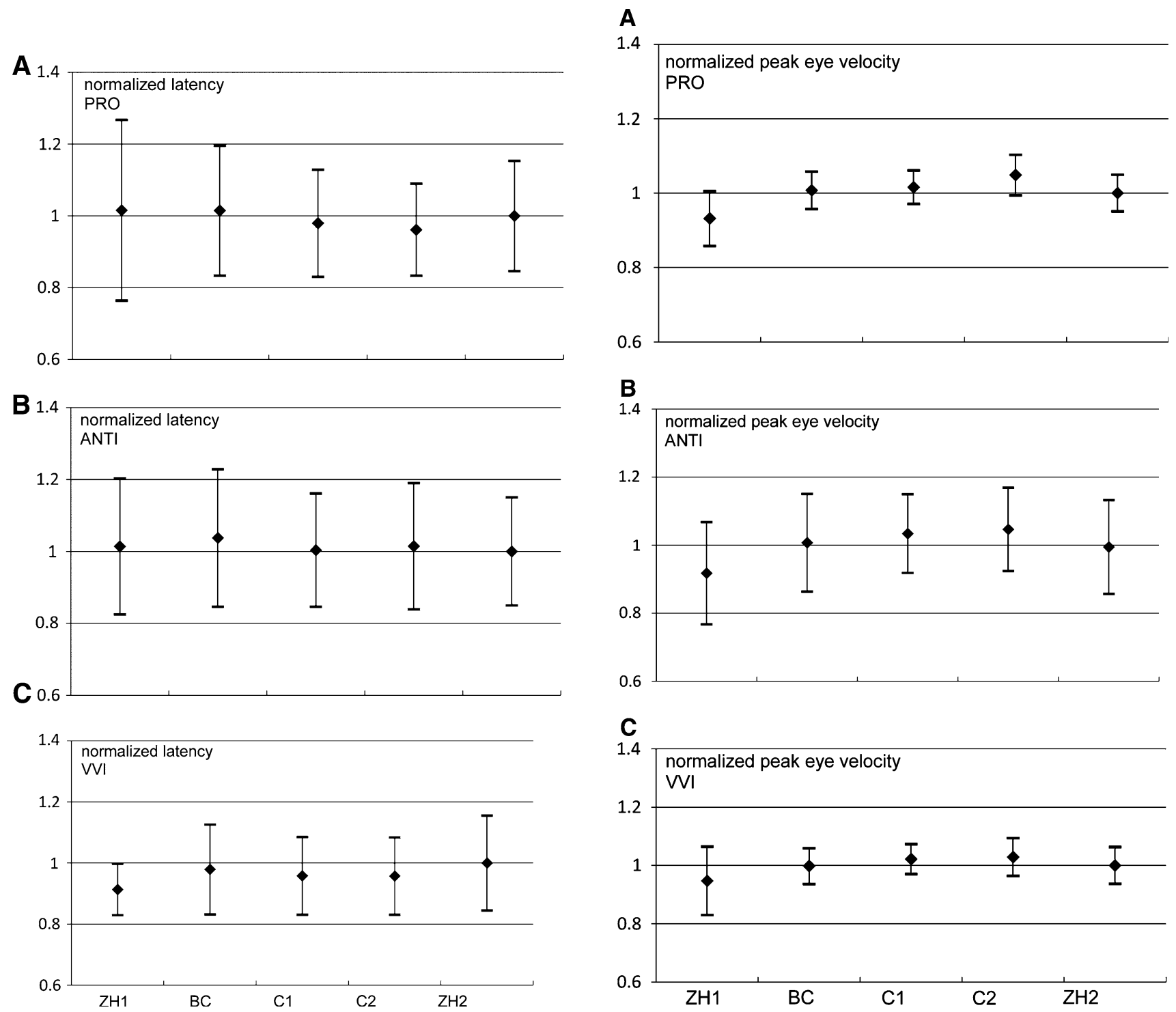

Fig. 5 Mean \pm SD of normalized latencies for each paradigm at each altitude of subgroup 2

Fig. 6 Mean $\pm \mathrm{SD}$ of normalized saturation velocities for each paradigm at each altitude of subgroup 2 


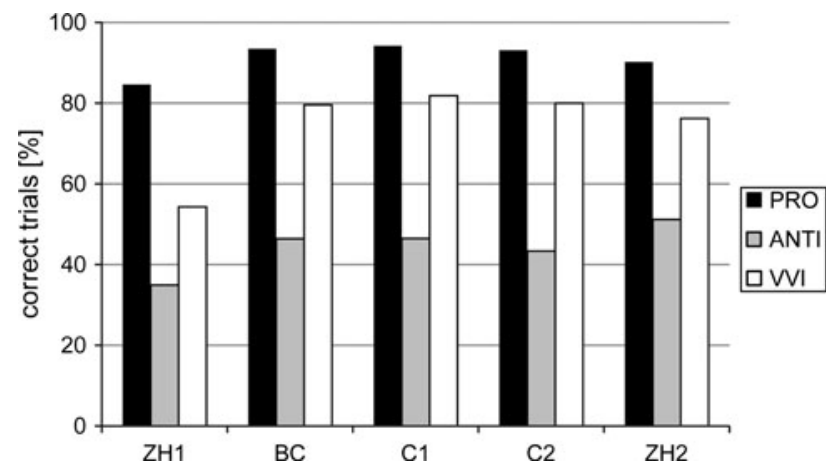

Fig. 7 Mean \pm SD percentage of correct trials for each paradigm at each altitude of subgroup 2

saccades with latencies of less than $100 \mathrm{~ms}$ ), or activation of an auxiliary or corollary network, which would not perform as precisely and thus lead to post-saccadic errors (Leigh and Zee 1999; Gaymard et al. 1998; Pierrot-Deseilligny et al. 2004). In addition, attention deficits (particularly due to parietal and/or frontal lobe deficits) would manifest themselves in the same way, as the attention system shares a multitude of cortical networks with the optomotor system (Nobre et al. 2000; Corbetta et al. 1998). Changes in the extrapyramidal system, which is also very vulnerable to hypoxic trauma, would produce frequent saccadic intrusions due to lack of control of the superior colliculus, which governs various saccade generators in the brainstem (Weber et al. 2000). Cerebellar and/or cerebellopontine lesions would quickly produce noticeable problems with the post-saccadic stability, e.g., gaze-evoked nystagmus. While the analysis of eye movements will easily be able to detect deficits due to global hypoxia, single lesions caused by other events, such as a small focal ischemia, may not always produce typical eye movement patterns or typical neuropsychological findings.

The assessment of the climbers using conventional neuropsychological test did not reveal any significant correlation of changes of cognitive function to the severity of hypoxia measured by pulse-oximetric $\mathrm{SaO}_{2}$. We did find a negative correlation with AMS-c scores (but not LLS scores) for the $2 / 7$ letters and the RFFT time to completion, thus the performance improved significantly in those climbers with more AMS symptoms. Similarly, the bisection precision was significantly better in the last test of the series and there was a significant decrease in time to complete $2 / 7$ letters at higher altitudes. Nevertheless, none of the neuropsychological test results revealed any evidence for impairment of cognitive function in hypoxic conditions. We assume that the significant increases in performance seen in the altitude measurements of some of the test are attributable to retest bias. In the context of standard neuropsychological assessments, test-retest reliability can be influenced due to practice effects related to both declarative (e.g., remembering the actual items on the tests) and procedural (e.g., remembering how to do the test) memory. Novelty effects (during the initial evaluation a test is novel to the subject, on repeat testing, the test becomes familiar) can increase or decrease performance (Suchy et al. 2010). Test-retest reliability can also be influenced by the retest interval (Heilbronner et al. 2010) and by floor and ceiling effects (Duff 2012). The applied neuropsychological tests are reported to have a comparatively high test-retest reliability (Basso et al. 1999) and our subjects were familiarized with the test procedures to reduce test-retest bias. Nevertheless, longitudinal withinsubject reliability is considerably higher in eye movement

Table 3 Statistical results of neuropsychological tests

\begin{tabular}{|c|c|c|c|c|c|}
\hline Test & Parameter & Corr. to altitude & Corr. to $\mathrm{SaO}_{2}$ & Corr. to LLs & Corr. to AMS-c \\
\hline LB PD & Ratio & $p<0.001^{*}$ & Ns & Ns & Ns \\
\hline \multirow[t]{2}{*}{$2 / 7$ numbers } & Time & Ns & Ns & Ns & Ns \\
\hline & Errors & Ns & Ns & Ns & Ns \\
\hline \multirow[t]{2}{*}{ 2/7 letters } & Time & $p<0.001^{*}$ & Ns & Ns & $p=0.04^{\dagger}$ \\
\hline & Errors & Ns & Ns & Ns & Ns \\
\hline \multirow[t]{2}{*}{ RFFT } & Time & Ns & Ns & Ns & $p=0.01^{\dagger}$ \\
\hline & Repetitions & Ns & Ns & Ns & Ns \\
\hline CT & Time & Ns & Ns & Ns & Ns \\
\hline
\end{tabular}

Summary result of the neuropsychological tests performed in $\mathrm{ZH} 1, \mathrm{BC}, \mathrm{C} 1, \mathrm{C} 2$, and $\mathrm{ZH} 2$

$\mathrm{SaO}_{2}$ arterial oxygen saturation; $L L s$ acute mountain sickness (AMS) assessed by applying the Lake Louise AMS protocol; $A M S$ - $c$ AMS assessed by applying the cerebral score of the environmental symptoms questionnaire; $L B P D$ mean deviation of the attempted bisection from the true centre of each line in percentage of the line length in line bisection test; 2/7 numbers 2 and 7 cancellation test applying numbers as distracters; $2 / 7$ letters 2 and 7 cancellation test applying letters as distracters; RFFT Ruff figural fluency test; $C T$ chess test

* Significant decrease in time needed to perform the test at different altitudes

$\uparrow$ Significant negative correlation to AMS-c score 
assessments (Wilson et al. 1993). This underlines our hypothesis, that standard neuropsychological testing might not be the optimal method to repeatedly assess cognitive function in the context of a high-altitude expedition-as outlined in the introduction.

Our study has a number of strengths and weaknesses. Despite substantial technical and logistical difficulties we were able to conduct a prospective investigation of cognitive performance in a large group of climbers during and after exposure to extreme altitudes. The high altitude of the camps and the summit did lead to very significant hypoxia and to a substantial number of participants suffering from altitude-related illnesses. As reported elsewhere, the incidence of suspected, clinically not apparent brain edema was high in the examined subjects (Bosch et al. 2008). Thus, the fact that we did not find any impairment of cognitive performance in our subjects cannot be attributed to insufficiently severe hypoxic conditions. The examined group consisted of amateur and not elite climbers of all age groups - in essence representing the growing population of recreational high-altitude sojourners.

The main study limitations were caused by the logistical restrictions of the research facilities at high altitudes. Data recording for the eye movement studies required $30 \mathrm{~min}$ per subject and altitude and was vulnerable to the unreliable power supplied by generators. In addition, due to adverse weather conditions, not all subjects reached the high-altitude research facilities in time to complete all planned eye movement recordings. However, we were able to record the majority of planned studies in each subject. The data sets before and after the expedition (ZH1 and $\mathrm{ZH} 2$ ) were complete, which underlines the validity of our findings excluding long-term cognitive impairment. This is a very important result as long-term cognitive impairment would clearly mandate to discourage climbers to attempt summits of high altitude. In 24 climbers $\mathrm{ZH} 1$ and $\mathrm{ZH} 2$ measurements of eye movements as well as at least two altitude measurements were completed. Complete data sets for all stimulus conditions on all altitudes were obtained only in the ten subjects in group S3. However, a true and systematic optomotor deficit would have produced a very clear, persistent, and well-known pathological pattern in the eye movement parameters. Such a deficit would have been detectable even when analyzing eye movement performance in the group S2 with missing values or in a small group of subjects such as the ten in group S3. Detectable impairment of cognitive function occurring during the climb would indicate a markedly increased risk for climbing-related incidents.

The variable individual reactions to environmental stress factors such as low ambient temperature, UV radiation, physical exercise, and mental stress could have influenced cognitive performance; these factors are difficult to measure and to correct for. However, we assume that environmental stress factors would impair rather than improve cognitive function. It therefore seems unlikely that a hypoxia-induced reduction in cognitive performance was masked by the influence of other, non-measured factors. Due to diligent acclimatization tactics and constant monitoring of all subjects and staff exposed to hypoxic conditions, we were able to avoid any occurrence of severe AMS or clinically apparent HACE. Our findings thus cannot exclude the possibility of cognitive impairment or longterm cerebral damage occurring after such an event.

We conclude that severe hypobaric hypoxia is well tolerated in healthy high-altitude climbers, without any indication of acute or long-term impairment of cognitive functions. Furthermore, we demonstrated that the investigation of eye movements is feasible during high-altitude expeditions and could represent a valuable tool for the study of human cognitive function under a variety of environmental conditions.

Acknowledgments We are thankful to the 32 volunteers who participated in our studies during the expedition and the following members of the Swiss Muztagh Ata Expedition Team: Timothy Holmes, Gregor Schubiger, and Frédéric Truffer. Furthermore, we appreciate the efforts of Kari Kobler and his Swiss Mountain guides, as well as the porters from Pakistan, China and Tibet. We thank Jeannie Wurz for editorial help with the manuscript.

Conflict of interest The authors declare that they have no conflict of interest.

\section{References}

Abraini JH, Bouquet C, Joulia F, Nicolas M, Kriem B (1998) Cognitive performance during a simulated climb of Mount Everest: implications for brain function and central adaptive processes under chronic hypoxic stress. Pflugers Arch 436(4): 553-559

Anooshiravani M, Dumont L, Mardirosoff C, Soto D, Delavelle J (1999) Brain magnetic resonance imaging (MRI) and neurological changes after a single high altitude climb. Med Sci Sports Exerc 31(7):969-972

Bahill AT, Stark L (1975) Overlapping saccades and glissades are produced by fatigue in the saccadic eye movement system. Exp Neurol 48(1):95-106. doi:10.1016/0014-4886(75)90225-3

Barton JJ, Jama A, Sharpe JA (1995) Saccadic duration and intrasaccadic fatigue in myasthenic and nonmyasthenic ocular palsies. Neurology 45(11):2065-2072

Basso MR, Bornstein RA, Lang JM (1999) Practice effects on commonly used measures of executive function across twelve months. Clin Neuropsychol 13(3):283-292. doi:10.1076/clin. 13.3.283.1743

Becker W (1989) Metrics. In: Wurtz RH, Goldberg ME (eds) The neurobiology of saccadic eye movements. Reviews of oculomotor research, vol 3, 1. Elsevier, New York, pp 13-67

Bosch MM, Barthelmes D, Merz TM, Bloch KE, Turk AJ, Hefti U, Sutter FK, Maggiorini M, Wirth MG, Schoch OD, Landau K (2008) High incidence of optic disc swelling at very high 
altitudes. Arch Ophthalmol 126(5):644-650. doi:10.1001/archopht. 126.5.644

Boxer AL, Garbutt S, Rankin KP, Hellmuth J, Neuhaus J, Miller BL, Lisberger SG (2006) Medial versus lateral frontal lobe contributions to voluntary saccade control as revealed by the study of patients with frontal lobe degeneration. J Neurosci 26(23):63546363

Braun JG, Kirk A (1999) Line bisection performance of normal adults: two subgroups with opposite biases. Neurology 53(3): $527-532$

Collewijn H, van der Mark F, Jansen TC (1975) Precise recording of human eye movements. Vision Res 15(3):447-450

Corbetta M, Akbudak E, Conturo TE, Snyder AZ, Ollinger JM, Drury HA, Linenweber MR, Petersen SE, Raichle ME, Van Essen DC, Shulman GL (1998) A common network of functional areas for attention and eye movements. Neuron 21(4):761-773. doi: 10.1016/S0896-6273(00)80593-0

Duff K (2012) Evidence-based indicators of neuropsychological change in the individual patient: relevant concepts and methods. Arch Clin Neuropsychol 27(3):248-261. doi:10.1093/ $\operatorname{arclin} / \operatorname{acr} 120$

Fayed N, Modrego PJ, Morales H (2006) Evidence of brain damage after high-altitude climbing by means of magnetic resonance imaging. Am J Med 119(2):168e161-166. pii:S0002-9343(05) 00674-1

Fischer MH (2001) Cognition in the bisection task. Trends Cogn Sci 5(11):460-462

Fransson PA, Patel M, Magnusson M, Berg S, Almbladh P, Gomez S (2008) Effects of 24-hour and 36-hour sleep deprivation on smooth pursuit and saccadic eye movements. J Vestib Res 18(4): 209-222

Garrido E, Castello A, Ventura JL, Capdevila A, Rodriguez FA (1993) Cortical atrophy and other brain magnetic resonance imaging (MRI) changes after extremely high-altitude climbs without oxygen. Int J Sports Med 14(4):232-234

Gaymard B, Ploner CJ, Rivaud S, Vermersch AI, Pierrot D (1998) Cortical control of saccades. Exp Brain Res 123(1-2):159-163

Green JF, King DJ (1998) The effects of chlorpromazine and lorazepam on abnormal antisaccade and no-saccade distractibility. Biol Psychiatry 44(8):709-715. pii:S0006-3223(97)00452-6

Heilbronner RL, Sweet JJ, Attix DK, Krull KR, Henry GK, Hart RP (2010) Official position of the American Academy of Clinical Neuropsychology on serial neuropsychological assessments: the utility and challenges of repeat test administrations in clinical and forensic contexts. Clin Neuropsychol 24(8):1267-1278. doi: $10.1080 / 13854046.2010 .526785$

Heitger MH, Jones RD, Anderson TJ (2008) A new approach to predicting postconcussion syndrome after mild traumatic brain injury based upon eye movement function. Conf Proc IEEE Eng Med Biol Soc 2008:3570-3573. doi:10.1109/IEMBS.2008. 4649977

Hornbein TF, Townes BD, Schoene RB, Sutton JR, Houston CS (1989) The cost to the central nervous system of climbing to extremely high altitude. N Engl J Med 321(25):1714-1719

Ilg UJ, Jin Y, Schumann S, Schwarz U (2006) Preparation and execution of saccades: the problem of limited capacity of computational resources. Exp Brain Res 171(1):7-15. doi: 10.1007/s00221-005-0255-z

Jason GW, Pajurkova EM, Lee RG (1989) High-altitude mountaineering and brain function: neuropsychological testing of members of a Mount Everest expedition. Aviat Space Environ Med 60(2): 170-173

Lebedev S, Van Gelder P, Tsui WH (1996) Square-root relations between main saccadic parameters. Invest Ophthalmol Vis Sci 37(13):2750-2758
Leigh RJ, Kennard C (2004) Using saccades as a research tool in the clinical neurosciences. Brain 127(Pt 3):460-477. doi:10.1093/ brain/awh035

Leigh RJ, Zee DS (1999) The neurology of eye movements. Contemporary Neurology Series. Oxford University Press, New York

Lieberman P, Protopapas A, Kanki BG (1995) Speech production and cognitive deficits on Mt. Everest. Aviat Space Environ Med 66(9):857-864

Masson GS, Mestre DR, Martineau F, Soubrouillard C, Brefel C, Rascol O, Blin O (2000) Lorazepam-induced modifications of saccadic and smooth-pursuit eye movements in humans: attentional and motor factors. Behav Brain Res 108(2):169-180

Munoz DP, Everling S (2004) Look away: the anti-saccade task and the voluntary control of eye movement. Nat Rev Neurosci 5(3):218-228

Nicolas M, Thullier-Lestienne F, Bouquet C, Gardette B, Gortan C, Richalet JP, Abraini JH (2000) A study of mood changes and personality during a 31-day period of chronic hypoxia in a hypobaric chamber (Everest-Comex 97). Psychol Rep 86(1):119-126

Nobre AC, Gitelman DR, Dias EC, Mesulam MM (2000) Covert visual spatial orienting and saccades: overlapping neural systems. Neuroimage 11(3):210-216. doi:10.1006/nimg.2000.0539

Pierrot-Deseilligny C, Rivaud S, Gaymard B, Agid Y (1991) Cortical control of reflexive visually-guided saccades. Brain 114(Pt 3): 1473-1485

Pierrot-Deseilligny C, Milea D, Muri RM (2004) Eye movement control by the cerebral cortex. Curr Opin Neurol 17(1):17-25

Regard M, Oelz O, Brugger P, Landis T (1989) Persistent cognitive impairment in climbers after repeated exposure to extreme altitude. Neurology 39(2 Pt 1):210-213

Ruff RM, Niemann H, Allen CC, Farrow CE, Wylie T (1992) The Ruff 2 and 7 selective attention test: a neuropsychological application. Percept Mot Skills 75(3 Pt 2):1311-1319

Ruff RM, Allen CC, Farrow CE, Niemann H, Wylie T (1994) Figural fluency: differential impairment in patients with left versus right frontal lobe lesions. Arch Clin Neuropsychol 9(1):41-55

Sampson JB, Cymerman A, Burse RL, Maher JT, Rock PB (1983) Procedures for the measurement of acute mountain sickness. Aviat Space Environ Med 54(12 Pt 1):1063-1073

Schenkenberg T, Bradford DC, Ajax ET (1980) Line bisection and unilateral visual neglect in patients with neurologic impairment. Neurology 30(5):509-517

Schmitt KU, Muser MH, Lanz C, Walz F, Schwarz U (2007) Comparing eye movements recorded by search coil and infrared eye tracking. J Clin Monit Comput 21(1):49-53. doi:10.1007/ s10877-006-9057-5

Schwarz U, Miles FA (1991) Ocular responses to translation and their dependence on viewing distance. I. Motion of the observer. J Neurophysiol 66(3):851-864

Schwarz U, Baumann K, Ilg UJ (2010) Influence of global motion onset on goal-directed eye movements. NeuroReport 21(7):479-484. doi:10.1097/WNR.0b013e328337badd

Spooner JW, Baloh RW (1979) Eye movement fatigue in myasthenia gravis. Neurology 29(1):29-33

Suchy Y, Kraybill ML, Franchow E (2010) Practice effect and beyond: reaction to novelty as an independent predictor of cognitive decline among older adults. J Int Neuropsychol Soc 17(1):101-111. doi:10.1017/S135561771000130X

Virues-Ortega J, Buela-Casal G, Garrido E, Alcazar B (2004) Neuropsychological functioning associated with high-altitude exposure. Neuropsychol Rev 14(4):197-224

Vorstius C, Radach R, Lang AR, Riccardi CJ (2008) Specific visuomotor deficits due to alcohol intoxication: evidence from 
the pro- and antisaccade paradigms. Psychopharmacology 196(2):201-210. doi:10.1007/s00213-007-0954-1

Weber B, Schwarz U, Kneifel S, Treyer V, Buck A (2000) Hierarchical visual processing is dependent on the oculomotor system. NeuroReport 11(2):241-247

West JB (1986) Do climbs to extreme altitude cause brain damage? Lancet 2(8503):387-388
Wilson SJ, Glue P, Ball D, Nutt DJ (1993) Saccadic eye movement parameters in normal subjects. Electroencephalogr Clin Neurophysiol 86(1):69-74

Yang Q, Wang T, Su N, Xiao S, Kapoula Z (2012) Specific saccade deficits in patients with Alzheimer's disease at mild to moderate stage and in patients with amnestic mild cognitive impairment. Age (Dordr). doi:10.1007/s11357-012-9420-z 